\title{
La insuficiencia de conocimientos para el diagnóstico microscópico de la malaria en técnicos de la red de laboratorios de Luanda, Angola
}

\author{
García Nazaré-Pembele ${ }^{1}$, Lázara Rojas², Fidel Ángel Núñez ${ }^{2}$ \\ 1 Instituto Nacional de Salud Pública, Luanda, República de Angola \\ 2 Departamento de Parasitología, Instituto de Medicina Tropical "Pedro Kourí", La Habana, Cuba \\ Introducción. La malaria, o paludismo, continúa siendo uno de los más importantes problemas de salud \\ pública a nivel mundial. La gota gruesa sigue siendo la técnica de referencia para su diagnóstico. \\ Objetivo. Evaluar los conocimientos para el diagnóstico de la malaria de técnicos del sector de la salud \\ en Luanda, Angola, mediante una encuesta. \\ Materiales y métodos. La preparación de la encuesta transcurrió en tres etapas: primero, se hicieron \\ entrevistas abiertas a los técnicos en torno a los diferentes procedimientos para el diagnóstico \\ microscópico de la malaria; en una segunda etapa, se preparó el cuestionario preliminar, y en la \\ tercera, se elaboró el cuestionario definitivo. La encuesta se hizo en un grupo de 120 técnicos de la \\ red nacional de salud entre los meses de abril y julio de 2013. Se comparó la proporción de respuestas \\ correctas e incorrectas considerando como significativo un valor de $p<0,05$. \\ Resultados. Las respuestas incorrectas predominaron sobre las correctas, de forma estadísticamente \\ significativa $(\mathrm{p}<0,05)$, en las preguntas sobre: las manifestaciones clínicas (68 Vs. 52$)$; las especies de \\ Plasmodium según zonas geográficas (76 Vs. 44); el tipo de granulaciones según la especie parasitaria \\ (96 Vs. 24); el tipo de lente para visualizar la gota gruesa (105 Vs. 15); la forma de hacer un informe del \\ resultado de la microscopía (76 Vs. 44); el tiempo y la concentración de las diferentes soluciones que \\ se utilizan (81 Vs. 39), y el número de parásitos por 200 leucocitos (96 Vs. 24). \\ Conclusiones. Se demostró la insuficiencia de conocimientos para el diagnóstico microscópico \\ de la malaria. Estos resultados servirán de base para el desarrollo de las herramientas de una \\ intervención educativa orientada a mejorar la calidad del diagnóstico de la malaria en la red de \\ laboratorios de Luanda.
}

Palabras clave: malaria/diagnóstico, recolección de datos, personal de laboratorio, Angola.

doi: http://dx.doi.org/10.7705/biomedica.v36i1.2623

Lack of knowledge regarding the microscopic diagnosis of malaria by technicians of the laboratory network in Luanda, Angola

Introduction: Malaria is still one of the most important public health problems worldwide. The diagnosis of this disease is still mainly based on thick blood films.

Objective: To evaluate the knowledge about malaria diagnosis of the technicians of the public health network in Luanda, Angola, by means of a survey.

Methods: This survey was carried out in three phases. In the first one, open interviews were done to technicians related with the different procedures for malaria diagnosis. In the second one, a preliminary questionnaire was prepared and evaluated. In the third phase, a definitive questionnaire was applied to 120 technicians from Luanda between April and July, 2013. The proportions of correct and incorrect answers were compared for every question of the survey.

Results: Significantly higher proportions of incorrect answers $(p<0.05)$ were found in the questions related to clinical manifestations, 68/52 $(p<0.05)$, species of Plasmodium according to geographical areas, 76/44 $(p<0.05)$, the type of granulations according to species, 96/24 $(p<0.01)$, the class of microscope magnifying glasses used to observe the thick smear, 105/15 $(p<0.01)$, the thick smear report, 76/44 $(p<0.01)$, the time and preparation of different stain solutions, $81 / 39(p<0.01)$, and the number of parasites counted per 200 leukocytes, 96/24 ( $p<0.01)$.

\footnotetext{
Contribución de los autores:

García Nazaré-Pembele: idea principal, trabajo de campo en la República de Angola y aplicación del cuestionario a los técnicos participantes

Lázara Rojas: preparación del cuestionario, interpretación de los resultados, escritura y revisión del trabajo

Fidel Ángel Núñez: diseño estadístico e interpretación de los resultados.
} 
Conclusions: Various failures for the microscopic diagnosis of malaria were observed amongst the evaluated technicians. These results will be useful as a baseline study before applying an educational intervention aimed to improve the quality of malaria diagnosis in Luanda's laboratory network.

Key words: malaria/diagnosis, data collection, laboratory personnel, Angola.

doi: http://dx.doi.org/10.7705/biomedica.v36i1.2623

La República de Angola es un país situado en la costa occidental de África, que presenta diferentes grados de endemismo de malaria o paludismo, enfermedad que es la principal causa de morbimortalidad en el país y afecta especialmente a niños menores de cinco años y mujeres embarazadas. En el 2013, el Programa Nacional de Control de la Malaria en Angola estimó una incidencia de seis millones de casos de malaria y tres mil muertes por esta causa (comunicación personal, Filomeno Fortes, jefe del Programa Nacional de Control de la Malaria en Angola).

En 1880, Alphonse Laveran publicó la primera descripción de los parásitos causantes de la malaria. Desde entonces, la observación en el microscopio de los parásitos que invaden los glóbulos rojos, con la ayuda de colorantes que tiñen el ADN parasitario, ha sido la técnica por excelencia para el diagnóstico del paludismo (1). La gota gruesa continúa siendo la técnica de referencia, sobre todo en los países con una gran morbimortalidad y una elevada intensidad de la transmisión. Esta técnica es de bajo costo y sencilla, y con ella se pueden establecer las densidades parasitarias y los estadios del ciclo de vida del parásito. Es un procedimiento sensible y específico que, si se realiza con la calidad requerida, brinda una gran confiabilidad $(2,3)$.

Los resultados de esta técnica dependen fundamentalmente de la experiencia del técnico microscopista, aunque existen otros factores que inciden en su utilización y afectan sensiblemente el éxito de los programas nacionales de control de la malaria en diferentes áreas endémicas.

En la guía del Programa Nacional de Control de la Malaria de Angola, se estipula que el tratamiento eficaz de la enfermedad debe basarse en un diagnóstico de laboratorio preciso que permita la identificación y la diferenciación de las especies de Plasmodium, dando lugar a un tratamiento

$\overline{\text { Correspondencia: }}$

García Nazaré, Departamento de Parasitología, Instituto de Medicina Tropical "Pedro Kourí", La Habana, Cuba

Teléfono: (53) 58296627

garciapembele@ipk.sld.cu y garciapembele@gmail.com

Recibido: 22/12/14; aceptado: 09/09/15 racional y esencial para retardar el fenómeno de la resistencia por el uso indiscriminado de fármacos antipalúdicos, para lo cual es necesario contar con personal bien entrenado y capacitado (4).

Según Fortes, et al. (4), en Angola la microscopía óptica, a pesar de ser la técnica de referencia para el diagnóstico de la malaria, presenta varias limitaciones, algunas de ellas asociadas a las condiciones propias de los laboratorios, y a la formación y la experiencia de los técnicos que hacen la lectura de la gota gruesa y el extendido.

El objetivo de esta investigación fue evaluar los conocimientos teóricos que pudieran estar incidiendo en la calidad del diagnóstico de la malaria mediante una encuesta a técnicos del sector de la salud en Luanda, Angola.

\section{Materiales y métodos}

La preparación del cuestionario transcurrió en tres fases. En la primera, se hicieron entrevistas abiertas a un grupo de técnicos considerados competentes dada su experiencia; las preguntas contenidas en esta entrevista se relacionaban con los diferentes procedimientos del diagnóstico de la malaria mediante el examen microscópico de la gota gruesa y del extendido de sangre periférica. Las entrevistas estuvieron a cargo de los investigadores del estudio.

Se establecieron varios grupos de preguntas que abarcaron los principales aspectos relacionados con el diagnóstico microscópico de la malaria y se dividieron por acápites, de la siguiente forma: aspectos generales de la malaria; aspectos morfológicos de las diferentes especies de parásitos que producen la enfermedad; aspectos de la microscopía propiamente dicha; aspectos de la coloración de la gota gruesa, y aspectos de los métodos de cuantificación de la parasitemia.

Para cada uno de los acápites se hicieron varios enunciados con diferentes opciones de respuestas (cuatro en total), de las cuales solo una era correcta.

El procesamiento y la evaluación de la información fueron sencillos, pues en cada grupo de preguntas solamente existían dos opciones en la respuesta: correcta o incorrecta. 
En la segunda fase se preparó el cuestionario preliminar. Con base en los resultados de las entrevistas, se estructuraron las preguntas de dicho cuestionario, a lo cual se agregó la experiencia acumulada en el Departamento de Parasitología del Instituto de Medicina Tropical "Pedro Kourí" en la preparación y la aplicación de instrumentos de este tipo, así como las opiniones sobre el tema de algunos especialistas en el empleo de las encuestas como herramientas de investigación. Además, se utilizaron los interrogantes clave planteados por la Organización Mundial de la Salud y la Organización Panamericana de la Salud en cursos relacionados con la malaria, dirigidos a estudiantes de laboratorio clínico (5). Después se revisó todo exhaustivamente, teniendo en cuenta que la encuesta podría servir para el establecimiento de líneas de base en la evaluación de una intervención posterior de capacitación del personal técnico a cargo del diagnóstico microscópico de la malaria.

En la tercera fase, se redactó el cuestionario definitivo: una vez elaborado el cuestionario preliminar, el cual contenía 25 preguntas, este se sometió al escrutinio de varios expertos. Posteriormente, se hizo la validación, es decir, se evaluó la factibilidad de su empleo mediante la aplicación del cuestionario a cuatro médicos especialistas en Parasitología y a otro grupo de 10 técnicos de laboratorio clínico familiarizados con el diagnóstico microscópico de la malaria, los cuales prestaban servicios fuera de las áreas donde se aplicó el cuestionario definitivo. Después de hacer las correcciones y las enmiendas resultantes de dichas consultas, el instrumento quedó listo para su utilización.

No se consideró necesaria la elaboración de un formato instructivo para la aplicación del cuestionario, pues esta estuvo a cargo de uno de los autores de la investigación.

El cuestionario definitivo incluyó 20 preguntas congregadas en cinco grupos. Uno constaba de cuatro preguntas $(20 \%)$ relacionadas con aspectos generales de la malaria, como la determinación de la forma más común de transmisión de la enfermedad, el reconocimiento de los síntomas clínicos más frecuentes, la producción del ciclo evolutivo, y otros aspectos generales sobre la epidemiología de la enfermedad. Otro grupo, también de cuatro preguntas (20\%), se refería a las características morfológicas de los parásitos causantes de la malaria y a las alteraciones más frecuentes que se producen en los glóbulos rojos según la especie parasitaria que los afecta. Un grupo de seis preguntas (30\%) versaba sobre los aspectos microscópicos propiamente dichos, incluidos los procedimientos para garantizar una óptima calidad en la toma de la muestra, los relacionados con el tipo de lente para visualizar la gota gruesa y los elementos del informe de los resultados del examen microscópico. Otro grupo de cuatro preguntas (20\%) se relacionaba con los métodos de coloración de la gota gruesa; por último, dos preguntas (10\%) del cuestionario se referían a los métodos de cuantificación de la parasitemia. El cuestionario definitivo recopiló, además, los datos generales de los participantes y se aplicó entre los meses de abril y julio de 2013 a un grupo de 120 técnicos de laboratorio de nivel básico y medio en el análisis clínico de la red nacional de salud de Luanda, República de Angola.

\section{Análisis estadístico}

Con el empleo el programa Epi-Info, versión 6.04 , se confeccionó una base de datos con la información obtenida de la encuesta a los 120 técnicos. El procesamiento y la evaluación de la información fueron sencillos, pues en cada grupo de preguntas solamente existían dos opciones en la respuesta: correcta o incorrecta.

Se calculó la proporción de respuestas correctas e incorrectas en cada una de las preguntas según los diferentes acápites y se compararon las frecuencias de ambos grupos para cada pregunta con una prueba de comparación de proporciones. Se consideró significativo un valor de $p<0,05$.

\section{Aspectos éticos}

Este estudio fue aprobado por la Comisión Científica del Hospital del Instituto de Medicina Tropical "Pedro Kourí" de La Habana, Cuba, y por el Comité de Ética del Instituto Nacional de Salud Pública de Luanda, Angola.

\section{Resultados}

De los 120 técnicos encuestados, todos respondieron las 20 preguntas del cuestionario y en ningún caso se anularon encuestas.

Como se muestra en el cuadro 1, al comparar la frecuencia de respuestas correctas e incorrectas en el grupo de preguntas que medían los conocimientos sobre aspectos generales de la malaria, en las relacionadas con las manifestaciones clínicas y las especies de Plasmodium según zonas geográficas, predominaron de forma significativa las respuestas incorrectas $(p<0,05)$. De igual forma, 
Cuadro 1. Comparación de la frecuencia de las respuestas correctas e incorrectas en cada pregunta de los cinco grupos de aspectos evaluados

\begin{tabular}{|c|c|c|c|c|}
\hline Aspectos evaluados & Total & $\begin{array}{c}\text { Respuestas } \\
\text { correctas } \\
\text { n (\%) }\end{array}$ & $\begin{array}{c}\text { Respuestas } \\
\text { incorrectas } \\
\mathbf{n}(\%)\end{array}$ & $\mathbf{p}$ \\
\hline \multicolumn{5}{|l|}{ Aspectos generales } \\
\hline La forma más común de transmisión de la malaria & 120 & $114(95,00)$ & $6(5,00)$ & $<0,001$ \\
\hline Manifestaciones clínicas de la malaria & 120 & $52(43,33)$ & $68(56,66)$ & $<0,05$ \\
\hline Ciclo evolutivo & 120 & $73(60,83)$ & $47(39,16)$ & $<0,001$ \\
\hline Especie de plasmodio según zona geográfica & 120 & $44(36,66)$ & $76(63,33)$ & $<0,001$ \\
\hline \multicolumn{5}{|l|}{ Morfología } \\
\hline Tipo de granulaciones según especie parasitaria & 120 & $24(20,00)$ & $96(80,00)$ & $<0,001$ \\
\hline Diferencias morfológicas de los gametocitos según especie parasitaria & 120 & $78(65,00)$ & $42(35,00)$ & $<0,001$ \\
\hline Elementos celulares fundamentales de Plasmodium & 120 & $86(71,66)$ & $34(28,33)$ & $<0,001$ \\
\hline Etiología del pigmento palúdico & 120 & $114(95,00)$ & $6(5,00)$ & $<0,001$ \\
\hline \multicolumn{5}{|l|}{ Microscopía } \\
\hline Relacionados con los glóbulos rojos parasitados & 120 & $68(56,66)$ & $52(43,33)$ & $<0,05$ \\
\hline Relacionados con los glóbulos blancos en una gota gruesa & 120 & $113(94,16)$ & $7(5,85)$ & $<0,001$ \\
\hline Relacionados con las dimensiones de una gota gruesa & 120 & $71(59,17)$ & $49(40,83)$ & $<0,001$ \\
\hline Relacionados con el tipo de lentes para visualizar la gota gruesa & 120 & $15(12,50)$ & $105(87,50)$ & $<0,001$ \\
\hline Elementos sanquíneos que pueden encontrarse en una gota gruesa & 120 & $86(71,66)$ & $34(28,33)$ & $<0,001$ \\
\hline Informe del resultado de la microscopía & 120 & $44(36,66)$ & $76(63,33)$ & $<0,001$ \\
\hline \multicolumn{5}{|l|}{ Coloración } \\
\hline Sustancias utilizadas para fijar el extendido de la sangre & 120 & $66(55,00)$ & $54(45,00)$ & $=0,121$ \\
\hline pH óptimo de las soluciones & 120 & $111(92,50)$ & $9(7,50)$ & $<0,001$ \\
\hline Tipo de marcador utilizado para identificar una gota gruesa & 120 & $53(44,16)$ & $67(55,83)$ & $=0,07$ \\
\hline Tiempo y concentración de las diferentes soluciones utilizadas & 120 & $39(32,50)$ & $81(67,50)$ & $<0,001$ \\
\hline \multicolumn{5}{|l|}{ Cuantificación } \\
\hline Número de parásitos por $\mu l$ de sangre & 120 & $71(59,16)$ & $49(40,83)$ & $<0,001$ \\
\hline Número de parásitos por 200 leucocitos & 120 & $24(20,00)$ & $96(80,00)$ & $<0,001$ \\
\hline
\end{tabular}

en relación con las preguntas sobre los aspectos morfológicos de Plasmodium spp., se pudo apreciar que las respuestas incorrectas sobre el tipo de granulaciones según la especie parasitaria, predominaron sobre las incorrectas de forma estadísticamente significativa $(p<0,01)$.

En el acápite concerniente a la microscopía, en lo relativo al tipo de lente para examinar la gota gruesa y la forma de hacer un informe correcto del resultado de la microscopía, también predominaron las respuestas incorrectas $(p<0,01)$, al igual que en las preguntas sobre los aspectos relativos a la coloración, específicamente el tiempo y la concentración de las diferentes soluciones utilizadas $(p<0,01)$.

Por último, en las preguntas sobre la cuantificación de la parasitemia, se observó que $80 \%$ de los encuestados respondió erróneamente a la pregunta relacionada con el número de parásitos por 200 leucocitos $(p<0,01)$.

\section{Discusión}

Los conocimientos sobre la malaria han experimentado significativos progresos durante los últimos decenios, con implicaciones en la práctica médica. Dichos avances en el conocimiento de la parasitosis aún no están incorporados al quehacer de los técnicos directamente vinculados con su diagnóstico. Uno de los conocimientos más arraigados, emanados de la práctica médica tradicional, se relaciona con las manifestaciones clínicas de la malaria aguda no complicada, caracterizada por el paroxismo palúdico: escalofríos, fiebre y sudoración (6). A pesar de esto, un número significativo de encuestados respondió erróneamente a la pregunta sobre las manifestaciones clínicas de la enfermedad, ya que atribuyeron a la malaria otros síntomas que guardan muy poca o ninguna relación con el paroxismo agudo de una malaria no complicada. Esta pudiera ser una de las causas de los falsos diagnósticos positivos que se emiten con cierta frecuencia en áreas endémicas de paludismo, pues el personal técnico no médico es el que en muchas ocasiones recibe a los pacientes y es responsable de su tratamiento y seguimiento.

Los aspectos epidemiológicos pueden contribuir positivamente a la correcta interpretación de un cuadro clínico palúdico. El conocimiento de las diferentes especies parasitarias predominantes según las áreas geográficas, en ocasiones, ayuda a la compleja identificación de las especies, lo 
que complementa la interpretación microscópica cuando esta es responsabilidad de personal no experimentado (6); sin embargo, un significativo número de los encuestados no pudo identificar correctamente las especies.

En cuanto a la morfología, los principales errores se dieron en la respuesta a la pregunta sobre los diferentes tipos de granulaciones que se observan en el glóbulo rojo parasitado y que varían en su tonalidad según la especie de Plasmodium. Se sabe que el eritrocito parasitado puede sufrir deformaciones y presentar granulaciones rosadas, denominadas de Schüffner en Plasmodium vivax y $P$. ovale, de Maurer, en $P$. falciparum, y de Ziemann, en $P$. malariae, estas últimas, difíciles de observar. Sin embargo, el mayor porcentaje de respuestas incorrectas se produjo en las relacionadas con la sustitución de granulaciones por las mitocondrias, que constituye un dato biológico importante y constituye un error de conocimiento básico en torno a los detalles morfológicos de Plasmodium determinantes para establecer un buen diagnóstico microscópico.

Reyburn, Coleman y Milne (7-9) han señalado que, si la gota gruesa está bien preparada y coloreada, no debe haber dificultad en la visualización microscópica del parásito $\mathrm{y}$, por ende, en la identificación de la especie de Plasmodium. No obstante, es de destacar que, dado el gran pleomorfismo en las diferentes estructuras morfológicas de los parásitos que producen la malaria (cada especie y cada estadio morfológico difieren entre sí), el personal encargado del diagnóstico tiene que estar muy bien entrenado para evitar confusiones, e incluso así, en ocasiones se presentan ciertas dificultades que resultan en un diagnóstico equivocado. Además, la gota gruesa y el extendido pueden presentar algunos contaminantes ambientales como hongos, partículas de polvo, células vegetales y bacterias. Estos artefactos a veces capturan los colorantes durante el proceso de tinción, provocando confusión y dificultades en el diagnóstico $(7,9)$. Asimismo, los colorantes mismos pueden estar contaminados con algunas de estas estructuras e interferir con el proceso de coloración y, en consecuencia, con la visualización microscópica.

Llama la atención que en las preguntas sobre el tipo de lente que se debe utilizar y la forma de presentar el informe del resultado de la microscopía, predominaron de forma significativa las respuestas erróneas. Es muy importante insistir en que, si se quiere tener una imagen microscópica nítida, debe combinarse un ocular de 10X y un objetivo de 100X para lograr un aumento total de 1.000X, que es la norma a la cual se ajustan los microscopios compuestos clásicos (2) para evitar esfuerzos innecesarios y el cansancio del microscopista. En este sentido, los errores se debieron al desconocimiento de este enunciado, lo cual puede provocar la selección errónea de lentes para el examen microscópico.

Un correcto informe de la lectura de la gota gruesa debe incluir la especie de Plasmodium, el estadio morfológico y la parasitemia. Son estos precisamente los aspectos más importantes que debe conocer el médico para establecer un tratamiento adecuado y eficiente en un paciente con paludismo $(10,11)$.

La magnitud de la parasitemia, o densidad parasitaria, es un parámetro objetivo que permite estimar la intensidad de la infección, la que a su vez se relaciona con la gravedad de las manifestaciones clínicas (12). Existen varios métodos para determinar la densidad parasitaria, cuyo manejo exige que los técnicos estén bien capacitados y entrenados en el reconocimiento de los diferentes tipos de células y de componentes de la sangre, en especial, los leucocitos. Precisamente este fue otro de los parámetros en el que se encontró el mayor porcentaje de errores en las respuestas emitidas por los encuestados, pues no sabían cómo determinar la parasitemia según el número de parásitos por 200 leucocitos. Estos errores tienen gran peso en el desempeño de los técnicos, pues la cuantificación precisa de la parasitemia proporciona al clínico un dato objetivo para evaluar la intensidad de la infección palúdica, el resultado del tratamiento y la sensibilidad in vivo a los fármacos esquizonticidas sanguíneos.

En los aspectos evaluados relacionados con la coloración, el tiempo y la concentración de las diferentes soluciones utilizadas para la coloración de la gota gruesa, también predominaron las respuestas erróneas. El desconocimiento de estos procedimientos resulta en una mala coloración de las estructuras de los parásitos. Resultados similares se obtuvieron en el estudio de Ginorio, et al. (13), sobre el control de calidad del diagnóstico de paludismo en la provincia de Cienfuegos; los autores consideraron que las razones fundamentales de esas dificultades radicaron en la inexistencia de documentos escritos o manuales de procedimientos técnicos 
para el diagnóstico, así como en la falta de entrenamientos y actualizaciones sistemáticas del personal encargado de esta actividad.

Según Hemme, et al. (14), para lograr calidad en el diagnóstico microscópico de la malaria y mejorar competencias, resulta indispensable la capacitación periódica del recurso humano que desempeña esta función en países en desarrollo. Este planteamiento, de ser aplicado en Angola, permitiría obtener una mayor confiabilidad en el diagnóstico microscópico de la malaria. El desarrollo de estrategias para mejorar las competencias de los trabajadores de la salud relacionadas con el diagnóstico y el tratamiento del paludismo, es muy necesario no solo en Angola sino, probablemente, en otros países de la región (15).

En algunos países del continente africano, entre los cuales se destacan Etiopía (16), Zambia (17), Congo (18) y Uganda (19), también se han encontrado deficiencias en el diagnóstico de la malaria y en algunos se han desarrollado programas de intervención educativa con el objetivo de mejorar la calidad del diagnóstico de esta parasitosis en las respectivas redes de laboratorios, lo que constituye unas de las bases sustentables para su control en la región.

Un estudio de 122 centros de salud de Etiopía (11) indicó que, aunque la mayoría de los centros tenían una infraestructura y equipamientos básicos aceptables,para el diagnóstico microscópico de la malaria, había un problema significativo de suministro de materiales y reactivos de laboratorio, y no se programaban entrenamientos ni supervisiones de apoyo, una debilidad del sistema que contribuye al deterioro de la calidad de los servicios que se prestan.

Desde el punto de vista teórico, este estudio encontró una serie de insuficiencias de conocimiento en el personal responsable del diagnóstico microscópico de la malaria. Se deben crear mecanismos educativos estables que tengan la garantía del sistema de salud pública de Angola. Conjuntamente con la capacitación, debe procurarse el establecimiento de un sistema de gestión de la calidad bien ejecutado que complemente las acciones para, así, mejorar la competencia del personal técnico encargado del diagnóstico microscópico de la malaria.

Este constituye el primer paso de una serie de intervenciones que se deben realizar para mejorar aspectos relacionados con el diagnóstico de esta importante parasitosis en el país siguiendo las recomendaciones de la Organización Mundial de la Salud para la evaluación del desempeño, con la respectiva acreditación periódica de competencias, como parte de un proceso de mejoría que incluya un programa de entrenamiento continuo. Además, debe tenerse en cuenta que en el desempeño final de los microscopistas de Luanda, pudieron haber influido también otros factores, como el volumen de trabajo, la adecuación de las condiciones de trabajo, y la falta de equipos e insumos apropiados, entre otros aspectos que no fueron evaluados en el presente estudio y que deben ser motivo de futuras investigaciones.

Los resultados obtenidos en este estudio servirán de base para la aplicación de una posible intervención educativa dirigida a mejorar la calidad del diagnóstico de la malaria en la red de laboratorios de Luanda, así como su posible extensión a todo el país.

\section{Conflicto de intereses}

Los autores declaran no tener ningún conflicto de intereses.

\section{Financiación}

Los recursos necesarios para el desarrollo del presente estudio fueron aportados por el Programa Nacional de Control de la Malaria y el Laboratorio Nacional de Salud de Luanda, República de Angola.

\section{Referencias}

1. Linder N, Turkki R, Walliander $\mathbf{M}$, Mårtensson $\mathbf{A}$, Diwan $\mathrm{V}$, Rahtu E, et al. A malaria diagnostic tool based on computer vision screening and visualization of Plasmodium falciparum candidate areas in digitized blood smears. PLoS One. 2014;9:e104855. http://dx.doi.org/10.1371/ journal.pone.0104855

2. Organization Mondiale de la Santé. Techniques de base pour le diagnostic microscopique du paludisme. Guide du stagiaire. Partie II. Guide de l'instructeur. Genéve: OMS; 2010. p. 7-57.

3. Organization Mondiale de la Santé. Comité OMS d'Experts du paludisme. Dix-huitième rapport. Genéve: OMS; 2013. p. 8-17.

4. Fortes F, Dimbu R, Neto Z, Ferreira C, Saraiva N, Do Rosário VE, et al. Estudo comparativo entre microscopia óptica e PCR para identificação de Plasmodium spp. em crianças em Angola. Ciência e Tecnologia. 2011;2:17-27.

5. World Health Organization. Self-learning materials and modules for health workers. A guide for their development, utilization and evaluation. Geneva: WHO; 2013. p. 10-28.

6. Llop A, Valdés-Dapena M, Zuaso JL. Microbiología y Parasitología Médicas. Primera edición. Tomo 3. La Habana: Editorial Ciencias Médicas; 2001. p. 117-85. 
7. Reyburn H, Mbatia R, Drakeley C, Carneiro I, Mwakasungula E, Mwerinde O. Overdiagnosis of malaria in patients with febrile illness in Tanzania: A prospective study. BMJ. 2004;329:1212. http://dx.doi.org/10.1136/bmj. 38251.658229 .55

8. Coleman RE, Maneechai N, Rachaphaew N, Kumpitak C, Miller RS, Soyseng V, et al. Comparison of field and expert laboratory microscopy for active surveillance for asymptomatic Plasmodium falciparum and Plasmodium vivax in western Thailand. Am J Trop Med Hyg. 2002;67:141-4.

9. Milne L, Kyi MS, Chiodini PL, Warhurst DC. Accuracy of routine laboratory diagnosis of malaria in the United Kingdom. J ClinPathol. 1994;47:740-2.

10. Bailey JW, Williams J, Bain BJ, Parker-Williams J, Chiodini PL. Guideline: The laboratory diagnosis of malaria. General Haematology Task Force of the British Committee for Standards in Haematology. Br J Haematol. 2013;163:573-80. http://dx.doi.org/10.1111/bjh.12572

11. Abreha T, Alemayehu B, Tadesse Y, Gebresillassie S, Tadesse A, Demeke $L$, et al. Malaria diagnostic capacity in health facilities in Ethiopia. Malar J. 2014;13:292. http:// dx.doi.org/10.1186/1475-2875-13-292

12. Maklet MT, Palmer CJ, Agger AL. A review of practical techniques for the diagnosis of malaria. Ann Trop Med Parasitol. 1998;92:419-33.

13. Ginorio D, Ortega S, Rojas L, Marín H, Oviedo A. Control de la calidad del diagnóstico de paludismo en la provincia de Cienfuegos, Cuba. Rev Cubana Med Trop. 2004;56: 49-53.
14. Hemme F, Gay F. Internal quality control of the malaria microscopy diagnosis for 10 laboratories on the ThaiMyanmar border. Southeast Asian J Trop Med Public Health. 1998;29:529-36.

15. Rowe AK, de León GF, Mihigo J, Santelli AC, Miller NP, van-Dúnem P. Quality of malaria case management at outpatient health facilities in Angola. Malar J. 2009;8:275. http://dx.doi.org/10.1186/1475-2875-8-275

16. Hailegiorgis B, Girma S, Mezlaku S, Teshi T, Demeke L, Gebresellasie S, et al. Laboratory malaria diagnostic capacity in health facilities in five administrative zones of Oromia Regional State, Ethiopia. Trop Med Int Health. 2010;15:1449-57. http://dx.doi.org/10.1111/j.1365-3156. 2010.02646.x

17. Keating J, Miller JM, Bennett A, Moonga HB, Eisele TP. Plasmodium falciparum parasite infection prevalence from a household survey in Zambia using microscopy and a rapid diagnostic test: Implications for monitoring and evaluation. Acta Trop. 2009;112:277-82. http://dx.doi. org/10.1016/j.actatropica.2009.08.011

18. Mukadi P, Gillet P, Lukuka A, Atua B, Kahodi S, Lokombe $\mathbf{J}$, et al. External quality assessment of malaria microscopy in the Democratic Republic of the Congo. Malar J. 2011;10:308. http://dx.doi.org/10.1186/1475-2875-10-308

19. Kyabayinze DJ, Achan J, Nakanjako D, Mpeka B, Mawejje $\mathbf{H}$, Mugizi R, et al. Parasite-based malaria diagnosis: Are health systems in Uganda equipped enough to implement the policy? BMC Public Health. 2012;12:695. http://dx.doi. org/10.1186/1471-2458-12-695 